\title{
Exploring the complex belowground world: Soil Ecology- The Soil Food Webs and Their Ecological Functions
}

\author{
Yong-Guan Zhu \\ Institute of Urban Environment, Chinese Academy of Sciences, Xiamen 361021, China \\ (c) Higher Education Press 2020
}

Soil is the important foundation for survival and development of human beings. Currently, soil health is increasingly being threatened because human activities, through disturbance and emission of toxic chemicals, have severely changed the structure and functions of soil food webs. However, the research progress on soil ecology is slow, at least in part due to the extreme complexity of soil. To understand the relationship between aboveground and belowground systems is imperative to sustainable use of soil resource, which has become one of the important frontiers of soil ecology and related disciplines.

The book "Soil Ecology-The Soil Food Webs and Their Ecological Functions" (Fu et al., 2019) is a timely publication in soil science. In this book, Professor Shenglei $\mathrm{Fu}$ and his colleagues characterized the structure and functions of soil food web through an integrative and dynamic angle based on their own research, and they also reviewed the historical milestone events and most recent research progress on the structure and functions of soil food web. Firstly, they introduced the classification and modeling of soil food web, and then illustrated the role of soil food web in regulation and indication of soil health, at last explored the potential application of soil food web in ecosystem restoration and sustainable agriculture. The authors focused on the key scientific questions and future challenges in soil ecology rather than covering everything.

Soil is a multi-dimensional complex system of high heterogeneity, and therefore soil ecology is a multi-disciplinary science. The authors emphasize the importance of the application of ideas, methodologies and techniques adapted from different disciplines in soil ecology and beyond. Meanwhile, they consider that "the spatial-temporal patterns of key ecological processes" is particularly important in illustrating the general structure and functions of soil food web, thus to add new dimensions to eco-geography.

This book is a timely addition to soil ecology, and I believe it will facilitate more in-depth research that will likely lead to new breakthrough development in soil ecology, particularly in cracking "bottleneck constraints" in soil ecology. This book should be a good reference for graduate students, postdocs and young scientists in soil ecology.

\section{Reference}

Fu, S.L., Zhang W.X., Shao Y.H., Shi L.L., Liu Z.F., 2019. Soil EcologyThe Soil Food Webs and Their Ecological Functions. Beijing: Science Press. 